\title{
Toxicidad del petróleo diesel en el primer estadio larval de la centolla (Lithodes santolla) y del centollón (Paralomis granulosa)
}

\author{
and false southern king crab (Paralomis granulosa) \\ Oscar Amin y Laura Comoglio \\ Centro Austral de Investigaciones Cientificas (CADIC - CONICET) \\ CC92 (V9410BFD), Ushuaia, Tierra del Fuego, Argentina. \\ oamin@infovia.com.ar
}

Toxicity of diesel-oil in the first larval stage of the southern king crab (Lithodes santolla)

\begin{abstract}
Lithodes santolla (commonly named "centolla") and Paralomis granulosa ("centollón") are the most important commercial fisheries in Tierra del Fuego and diesel-oil represents one of the more often transported hydrocarbons into Ushuaia Bay. So that the aim of the present work was to evaluate the acute toxicity of the water soluble fraction of diesel oil (LC50-96h) on the first larval stage (zoea I) for both species and the effect on the molt (zoea I-II) of Lithodes santolla analyzing the molt success and the mean lifetime of the zoea I stage.

The results obtained (LC50-96 h) show that zoea I of $L$. santolla was more sensitive than $P$. granulosa $(\mathrm{p}<0.01)$. While at sub lethal concentrations the water soluble fraction of diesel oil affected the molt success, but not the mean lifetime of L. santolla zoea I. At higher concentrations than $29 \%$ no zoea I reached the next larval stage.

Others sublethal effects such as failure to swim after $24 \mathrm{~h}$ of exposure were observed in both species in concentrations higher than $13 \%$.
\end{abstract}

Key words: diesel-oil, Lithodes santolla, Paralomis granulosa, larval stage, toxicity

\section{Introducción}

La centolla Lithodes santolla (Molina, 1782) y el centollón Paralomis granulosa (Jacquinot, 1847) conforman desde hace más de tres décadas la principal pesquería en el sector argentino del Canal Beagle, Tierra del Fuego.

La distribución de la centolla abarca desde Chiloé en el Océano Pacífico, las aguas del Canal Beagle y por el Atlántico llega hasta las costas de Uruguay, mientras que el centollón se distribuye desde Paso Tenaún (Chile) hasta el golfo San Jorge (Argentina), incluyendo Canal Beagle, Cabo de Hornos e islas Malvinas (Macpherson 1988).
Resumen.- Teniendo en cuenta que Lithodes santolla (centolla) y Paralomis granulosa (centollón) constituyen una pesquería mixta de gran actividad económica en Tierra del Fuego y que el petróleo diesel representa uno de los combustibles de mayor transporte marítimo hacia la ciudad de Ushuaia, se evaluó la toxicidad aguda (CL50-96h) de la fracción soluble del mismo en el estadio zoea I de ambas especies y sobre la ecdisis (zoea I-II) en L. santolla evaluando el porcentaje de mudas exitosas y analizando el tiempo de vida medio del estadio zoea I registrado en cada tratamiento.

Los resultados obtenidos indican que las larvas zoea I de $L$. santolla resultaron significativamente más sensibles que las de $P$. granulosa en exposiciones agudas $(\mathrm{p}<0,01)$, mientras que a concentraciones subletales los tratamientos afectaron significativamente la proporción de mudas obtenidas, no así el tiempo de vida medio. No se obtuvieron zoea II en la exposición a concentraciones superiores a $29 \%$ de fracción soluble.

Se observaron otros parámetros subletales como fallas en la natación, las cuales se detectaron a las $24 \mathrm{~h}$ de exposición a concentraciones mayores al 13\% de fracción soluble.

Palabras clave: petróleo diesel, Lithodes santolla, Paralomis granulosa, larvas, toxicidad
El desarrollo larval de la centolla comprende tres estadios zoea con una duración total aproximada de 25 días en condiciones experimentales y un estadio postlarva (Campodónico 1971). En el caso del centollón el desarrollo larval consiste en dos estadios zoea que abarcan en condiciones experimentales, entre 18 y 20 días y una postlarva (Vinuesa et al. 1989).

La ciudad de Ushuaia ha incrementado su población y consecuentemente su actividad portuaria sensiblemente en los últimos 20 años. Anualmente, el puerto recibe cerca de 900 embarcaciones (turísticas, comerciales, pesqueras y de transporte de combustibles) las cuales además realizan operaciones de 
reabastecimiento de combustible en una estación costera de bahía Ushuaia. A pesar de los controles, en muchos casos la eliminación de combustible al agua es visualmente perceptible, aunque los niveles de hidrocarburos detectados en sedimentos costeros no resultan elevados (Commendatore \& Esteves 2001 ${ }^{1}$ ).

Si bien las pruebas de toxicidad aguda no pueden ser usadas para predecir directamente el riesgo ambiental, el uso más apropiado de las mismas es medir la toxicidad de las sustancias o la sensibilidad de diferentes especies a las sustancias, con el objeto de identificar poblaciones en riesgo y posibilitar el desarrollo de pruebas sobre factores que afectan parámetros subletales que comprometen la supervivencia de las poblaciones (Hansen 1984).

Teniendo en cuenta que los estadios larvales son, en general, los más sensibles a cambios ambientales y que todo el combustible empleado para transporte en la provincia ingresa por vía marítima a la ciudad de Ushuaia, el presente trabajo tiene como objetivo evaluar la toxicidad de la fracción soluble del petróleo (diesel) sobre la supervivencia del primer estadio larval de Lithodes santolla y Paralomis granulosa, dos especies de gran interés para la región.

\section{Materiales y Métodos}

Los organismos utilizados en los bioensayos fueron obtenidos a partir de un grupo de cinco hembras ovígeras por especie, recolectadas en el canal Beagle, durante agosto y septiembre del año 2000.

Se utilizó como tóxico de prueba combustible diesel para embarcaciones, procedente de la refinería YPF " $\mathrm{La}$ Plata", Buenos Aires, Argentina (densidad 0,83-0,85, punto de inflamación $62^{\circ} \mathrm{C}$ ). Se preparó una solución concentrada de la fracción soluble en agua (FSA), de acuerdo a la metodología descripta por Anderson et al. (1974) mezclando una proporción de 9 agua: 1 petróleo diesel, sometida a agitación mecánica durante 20 h, a partir de la cual se prepararon las correspondientes diluciones.

Las larvas nacidas durante una misma noche (edad menor a $24 \mathrm{~h}$ ) fueron separadas y seleccionadas de acuerdo al buen estado general y movilidad de las mismas.

\footnotetext{
1 Commendatore M \& JL Esteves. 2001. Hidrocarburos alifáticos en la zona costera de la Provincia de Tierra del Fuego (Argentina). Datos preliminares. Actas del IX Congreso Latinoamericano de Ciencias del Mar. San Andrés, Colombia
}

Para las pruebas de toxicidad aguda $(96 \mathrm{~h}$ de exposición) lotes de 30 organismos por tratamiento fueron distribuidos al azar en réplicas de 10 individuos cada una y expuestos a las siguientes concentraciones nominales: 6,$6 ; 10 ; 15 ; 22,5 ; 33,75 ; \quad 50,6 ; 75,9$ expresadas en porcentaje de fracción soluble de diesel en agua. Para analizar el efecto sobre la muda, lotes de 40 organismos (zoea I de L. santolla) por tratamiento fueron distribuidos aleatoriamente en réplicas de 10 individuos cada una y expuestos a las siguientes concentraciones nominales: 9, 13, 19 y 29 expresadas en porcentaje de fracción soluble de diesel en agua. Para cada bioensayo se incluyó el correspondiente control de agua.

Los bioensayos fueron desarrollados siguiendo las recomendaciones de Ward \& Parrish (1982) para sistemas estáticos con renovación de agua diaria. Las condiciones experimentales fueron las siguientes: temperatura del agua $7,5 \pm 0,5^{\circ} \mathrm{C}$ (mantenido en baño termostático), fotoperíodo $12 \mathrm{~h}$ luz: $12 \mathrm{~h}$ oscuridad y salinidad $32 \%$ o. El volumen del agua de dilución fue de $150 \mathrm{~mL}$ y durante los experimentos los recipientes permanecieron tapados y sin aireación. Diariamente los individuos fueron contabilizados, removiendo a los organismos muertos y registrando las mudas. El criterio de mortalidad utilizado fue la ausencia de latido cardíaco (Rodríguez \& Amin 1991, Wells \& Sprague 1976). Durante el transcurso de ambos experimentos las larvas no fueron alimentadas, régimen que no interfiere con su supervivencia debido al carácter lecitotrófico que presentan en este estadio de desarrollo (Comoglio \& Vinuesa 1991).

Tanto la CL50-96 h como el tiempo medio de duración del estadio zoea I fueron estimados de acuerdo al método de probit incluyendo la corrección de Abbott para el control de la mortalidad de los controles (Finney 1971). La comparación entre las CL50 fue realizada considerando estadísticamente significativas aquellas en las que el cociente CL50 mayor / CL50 menor excede el valor correspondiente al valor crítico establecido por APHA (1980). Los porcentajes de muda en cada tratamiento, registrado sólo para el caso de Lithodes santolla, fueron comparados mediante la prueba exacta de Fisher (Sokal \& Rolhf 1981).

\section{Resultados y Discusión}

La supervivencia de las larvas de cada especie a $96 \mathrm{~h}$ de exposición puede observarse en la Fig. 1. En todos los experimentos se obtuvo una mortalidad para los controles dentro de los niveles esperados de acuerdo a trabajos realizados en condiciones de cultivo (Comoglio \& Vinuesa op. cit.). Si bien durante las primeras $72 \mathrm{~h}$ no pudo estimarse analíticamente los valores 
correspondientes de CL50, se obtuvieron mortalidades levemente superiores al $50 \%$ en las concentraciones de 33,$75 ; 50,6$ y 75,9 para $L$. santolla y en 75,9 para $P$. granulosa.

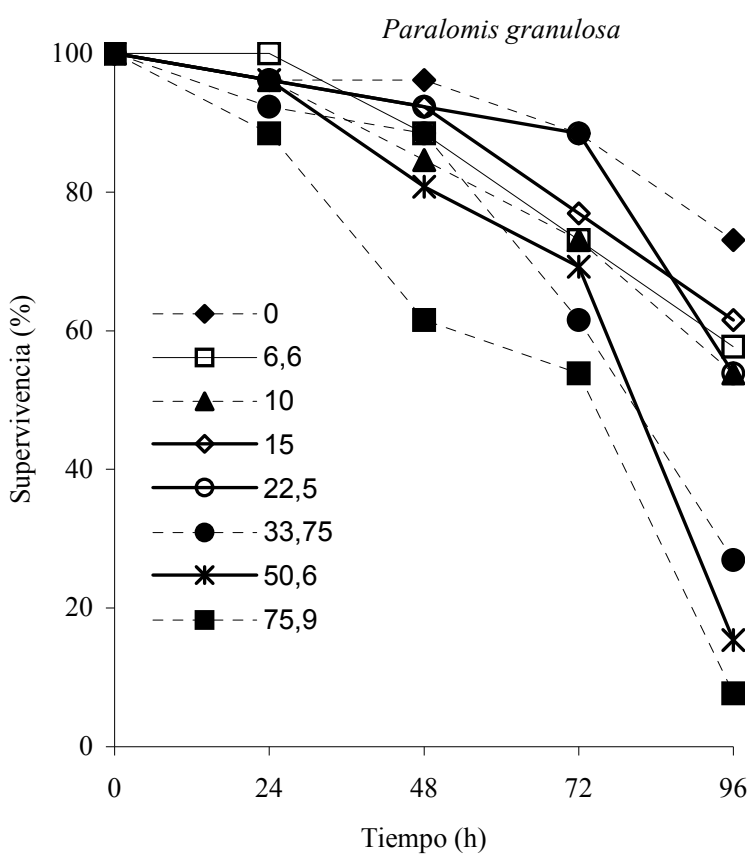

Los valores estimados de CL50 96 h se presentan en la Tabla 1. Se hallaron diferencias significativas $(\mathrm{p}<0,01)$ entre las larvas de las especies estudiadas, resultando L. santolla más sensible que $P$. granulosa.

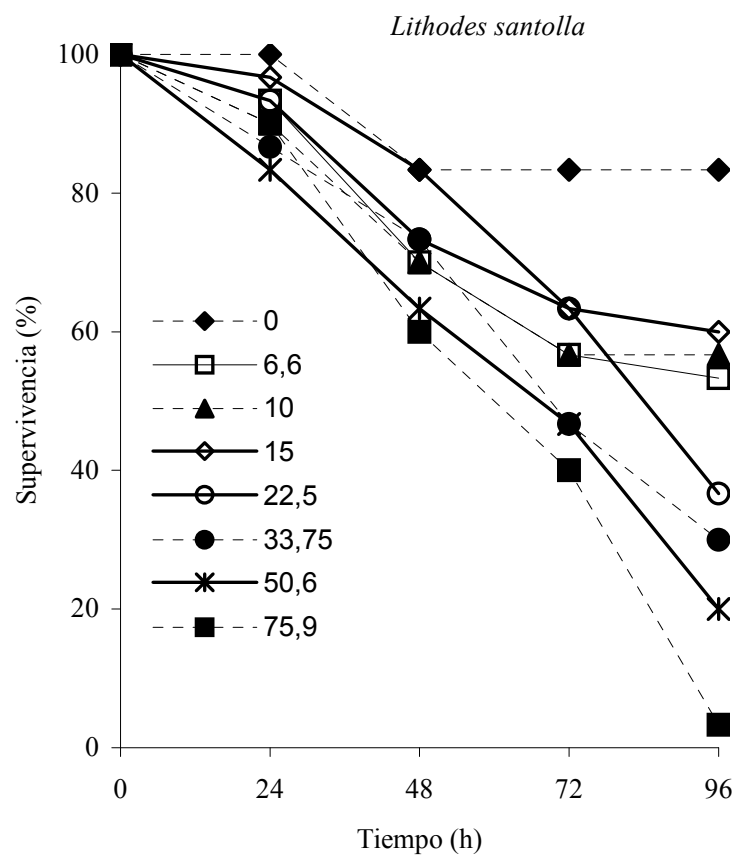

Figura 1

Porcentaje de supervivencia de larvas zoea I de Lithodes santolla y Paralomis granulosa expuestas a diferentes concentraciones de fracción soluble de petróleo diesel (expresadas en porcentaje)

Percentage of survival of zoea I larvae of Lithodes santolla and Paralomis granulosa exposed to different concentrations of water soluble fraction of diesel oil (expressed in percentage)

Tabla 1

Valores estimados de CL50-96 h e intervalos de confianza, expresados en porcentaje de fracción soluble en agua (FSA), pendiente y coeficiente de correlación de los parámetros del análisis de probit y comparación entre las CL50 para Lithodes santolla (Ls) Paralomis granulosa (Pg)

LC50-96 h values expressed in percentage of water soluble fraction (WSF), confidence limits, slope and correlation coefficient from probit analysis and comparison between LC50 for Lithodes santolla (Ls) and Paralomis granulosa (Pg)

\begin{tabular}{|c|c|c|c|c|}
\hline \multicolumn{1}{|c|}{ Especie } & $\begin{array}{c}\text { CL50-96 h } \\
\text { (Límite de confianza 95\%) }\end{array}$ & Pendiente & $\mathrm{R}^{2}$ & $\begin{array}{c}\text { Relación CL50 } \\
P g / L s\end{array}$ \\
\hline Paralomis granulosa (centollón) & $\begin{array}{c}27,57 \\
(19,67-36,15)\end{array}$ & 2,53 & 0,83 & $\begin{array}{c}1,48 \\
(\mathrm{p}<0,01)\end{array}$ \\
\hline Lithodes santolla (centolla) & $\begin{array}{c}18,64 \\
(13,01-24,62)\end{array}$ & 1,80 & 0,81 & \\
\hline
\end{tabular}




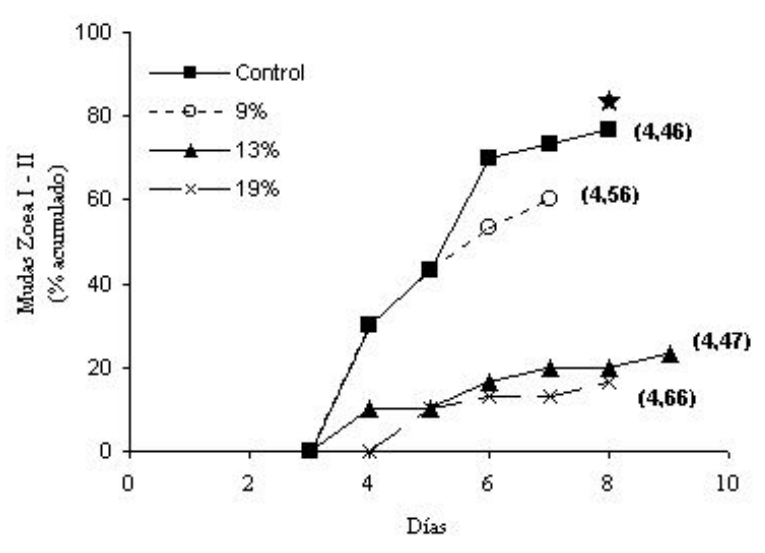

Figura 2

Porcentaje acumulado de mudas al estadio zoea II para larvas de Lithodes santolla expuestas a diferentes concentraciones de fracción soluble de petróleo diesel (expresadas en porcentaje). Los valores entre paréntesis indican el tiempo de vida medio. EL asterisco indica diferencias significativas

Cumulative percentage of molting from zoea I to zoea II of Lithodes santolla, exposed to different concentrations of water soluble fraction of diesel oil (expressed in percentage). Values between brackets indicate mean lifetime. Asterisk means significant differences

Los efectos de la exposición a la FSA sobre la muda en larvas zoea I de $L$. santolla pueden observarse en la Fig. 2. Se detectaron diferencias significativas, siendo el porcentaje de muda obtenido en el control mayor que los correspondientes a los tratamientos $(\mathrm{p}<0,01)$. Se obtuvo $76,6 \%$ de mudas exitosas en el control y $60 \%$; $23,3 \%$ y $16,7 \%$ en las concentraciones 9,13 y 19 respectivamente. A partir de $29 \%$ de fracción soluble no se obtuvieron mudas a zoea II. No se registraron diferencias en el tiempo medio en que las zoeas I alcanzaron el estadio subsiguiente.

Los hidrocarburos están formados por una numerosa mezcla de compuestos los cuales difieren en solubilidad, resultando importante considerar el acuerdo general que existe en la literatura acerca de la mayor toxicidad que poseen los productos refinados de los hidrocarburos sobre los crudos (La Roche et al. 1970, Anderson et al. 1974). El efecto tóxico del petróleo sobre los organismos se debe principalmente a los hidrocarburos presentes en la fase acuosa (Espina \& Vanegas 1996) y si bien es muy amplia la variedad de efectos descriptos en relación a esta toxicidad, al tratarse de tipos de derivados de petróleo particulares en cada región y de organismos de prueba locales, las comparaciones deben realizarse con las limitaciones y cuidados correspondientes.

En general, se sabe que en este tipo de bioensayos, las larvas de ambientes fríos son más sensibles que las de ambientes cálidos, debido a que a temperaturas bajas las fracciones solubles son tóxicas por períodos más prolongados dado que los procesos de evaporación y biodegradación son más lentos aumentando su persistencia en el medio (Cheatham et al. $1976^{2}$ ).

Para especies de aguas frías resulta particularmente interesante resaltar que sus desarrollos larvales son comparativamente más prolongados que las especies de aguas cálidas $\mathrm{y}$, en forma general, como sugieren Brodersen et al. (1977) las larvas de especies antárticas y subantárticas son especialmente más sensibles que los adultos debido a que mudan con mayor frecuencia.

Tomando en cuenta estas consideraciones y a pesar que los resultados de los bioensayos son limitados en el sentido de su extrapolación a los efectos en el ambiente, es importante señalar que la eventual presencia de hidrocarburos en el medio además de ser letal, aumenta la sensibilidad de las poblaciones al afectar procesos metabólicos y motrices que convierten a las larvas en potenciales presas vulnerables.

Efectos de otras sustancias sobre la mortalidad en larvas de L. santolla han sido estudiados por Lombardo et al. (1982, 1991). Estos autores además describen cualitativamente efectos subletales de los plaguicidas etil paratión (DNTP) y diclorovinilfosfato (DNVP) como disminución de la actividad natatoria, expansión del caparazón y rigidez del abdomen, mientras que para el lindano y la acetona se observaron retrasos en la muda. Algunos de estos efectos pueden considerarse inespecíficos ya que no sólo han sido observados en los citados casos y en el presente trabajo, sino también en la exposición a metales pesados y a petróleo crudo en larvas de ambas especies (Amin 1995).

Weis et al. (1992) describen como efecto general a la exposición a hidrocarburos la disminución en el incremento por muda. Retrasos en el desarrollo larval e inhibición en el crecimiento y muda en exposiciones a hidrocarburos también han sido reportados para larvas de numerosas especies de crustáceos, asociando estas respuestas a la interferencia de este tipo de compuestos sobre las vías metabólicas normales de los ácidos grasos y a disrupciones energéticas en general (Capuzzo \& Lancaster 1981).

En relación al presente trabajo donde el efecto observado para estimar la CL50 fue la ausencia del

\footnotetext{
${ }^{2}$ Cheatham DI, RE Milleman \& NE Stewart. 1976. Effects of temperature, volatility, and biodegradation on the persistence of aromatic hydrocarbon on seawater. NOAA-EPA Symposium on Fate and Effects of Petroleum Hydrocarbons, Seattle, Washington
} 
latido cardíaco, ha sido posible observar fallas en la natación como otro indicador de daño, tal como sugieren Carls \& Rice (1984) y Brodersen et al. (1977). En coincidencia con estos últimos autores, se percibieron para ambas especies estudiadas, problemas de movilidad en las larvas expuestas a concentraciones superiores a $13 \%$ de fracción soluble dentro de las primeras $24 \mathrm{~h}$ de exposición. Por otra parte, Morton \& $\mathrm{Wu}$ (1977) observaron una rápida pérdida en las habilidades natatorias de larvas nauplios de Balanus amphitrite amphitrite y Balanus variegatus variegatus expuestos a queroseno así como también Rice et al. $\left(1981^{3}\right)$ detectan la misma respuesta en larvas de "king crab" Paralithodes camtschatica, Cancer magister, Pandalus hypsinotus y Eualus suckleyi, luego de algunos minutos de exposición a la fracción soluble de petróleo crudo.

Como es discutido por Brodersen (1987) la primera manifestación de las larvas a exposiciones de la fracción soluble de hidrocarburos suele ser la finalización de la actividad natatoria, provocando un "retraso" en la muerte de los organismos. Este hecho debe ser especialmente atendido al momento de seleccionar el mejor parámetro de daño que describa el efecto tóxico de estas sustancias. La sensibilidad podría ser comparada en base a la morbilidad de los organismos más que a la mortalidad misma.

Si bien hasta el presente no se han hallado larvas de ambas especies en condiciones naturales, no se descarta que su habilidad natatoria les permitiría en ciertas circunstancias evadir los eventuales derrames de hidrocarburos. Para evaluar con mayor precisión su vulnerabilidad se debería conocer su distribución en el ambiente así como los efectos a largo plazo de la exposición a la fracción soluble del petróleo diesel a fin de aportar datos relevantes sobre el riesgo potencial de las poblaciones.

\section{Literatura citada}

American Public Health Association, American Water Works Association, Water Pollution. Control Federation. 1980. Standard methods for the examination of water and wastewater, 15th ed. American Public Health Association, Washington, D.C. 1134 pp.

3 Rice SD, S Korn, CC Brodersen, SA Lindsay \& SA Andrews. 1981. Toxicity of ballast-water treatment effluent to marine organisms at Port Valdez, Alaska. En: 1981 Oil Spill Conference Proceedings. Am. Pet. Inst. Washington DC, 742 pp.
Amin O. 1995. Toxicidad para invertebrados marinos de algunos metales pesados detectados en la zona costera próxima a Ushuaia, Tierra del Fuego. Tesis Doctoral. Facultad de Ciencias Exactas y Naturales. Universidad de Buenos Aires. 141 pp.

Anderson JW, JM Neff, BA Cox, HE Tatem \& GM Hightower. 1974. Characteristics of dispersions and water soluble extracts of crude and refined oils and their toxicity to estuarine crustaceans and fish. Marine Biology 27: 7588 .

Brodersen C, SD Rice, JW Short, TA Mecklienburg \& JF Karinen. 1977. Sensitivity of larval and adult Alaskan shrimp and crabs to acute exposure of the water soluble fraction of cook inlet crude oil. Proceedings of 1977 Oil Spill Conference, (Prevention, Behavior, Control Cleanup) American Petroleum Institute: 575-578.

Brodersen C. 1987. Rapid narcosis and delayed mortality in larvae of king crabs and kelp shrimp exposed to the watersoluble fraction of crude oil. Marine Environmental Research 22: 233-239.

Campodónico I. 1971. Desarrollo larval de la centolla Lithodes antarctica Jaquinot en condiciones de laboratorio (Crustacea Decapoda Anomura: Lithodidae). Anales del Instituto de la Patagonia 2 (1-2): 181-190.

Capuzzo JM \& BA Lancaster. 1981. Physiological effects of petroleum hydrocarbons on larval lobster (Homarus americanus): Hydrocarbon accumulation and interference with lipid metabolism. En: Vernberg J, A Calabrese, FP Thurberg \& WB Vernberg (eds). Biological Monitoring of Marine Pollutants: 477 - 499. Academic Press, Nueva York.

Carls MG \& SD Rice. 1984. Toxic contributions of specific drilling mud components to larval shrimp and crabs. Marine Environmental Research 12: 45-62.

Comoglio L \& J Vinuesa. 1991. Larval culture under laboratory conditions of southern king crab Lithodes santolla and false king crab Paralomis granulose. European Aquaculture Society. Special Publication No 15: 349-351.

Espina S \& C Vanegas. 1996. Ecotoxicología y Contaminación. En: Vazquez Botello A, JL Rojas Galviz, JA Benitez \& D Zarate Lomelí (eds). Golfo de Mexico, Contaminación e Impacto: Diagnóstico y Tendencias. EPOMEX Serie Científica 5: 69-106. Universidad Autónoma de Campeche, México.

Finney DJ. 1971. Probit analysis. 3rd ed. Cambridge University Press, Cambridge. 333 pp.

Hansen DJ. 1984. Utility of toxicity test to measure effects of substances on marine organisms. En: White HH (ed). Concepts in Marine Pollution Measurements. Chapter 1, Toxicity test: 33-56. Maryland Sea Grant Publications, University of Maryland, Maryland 
La Roche G, R Eisler \& CM Tarzwell. 1970. Bioassay procedures for oil and oil dispersant toxicity evaluation. Journal of Water Pollution Control Federation 42: 19821989.

Lombardo RJ, L Ferrari \& JH Vinuesa. 1982. Efectos del etil parathion y DDVP sobre larvas de centolla Lithodes antarticus Jaquinot. Ecosur (9) 18: 141-151.

Lombardo RJ, L Ferrari \& JH Vinuesa. 1991. Effects of lindane and acetone on the development of larvae of the southern king crab (Lithodes antarticus Jaquinot). Bulletin of Environmental Contamination and Toxicology 46:185192.

Macpherson E. 1988. Revision of the family Lithodidae Samouelle, 1819 (Crustacea, Decapoda Anomura) in the Atlantic Ocean. Monografías en Zoología Marina 2: 9153.

Morton B \& RSS Wu. 1977. The toxic effects of hydrocarbons upon the naupliar and adult stages of Balanus (Crustacea: Cirripedia). Marine Pollution Bulletin 8: 232-236.
Rodríguez E \& O Amin. 1991. Acute toxicity of Parathion and 2,4 D to larval and juvenile stage of Chasmagnathus granulata (Decapoda, Brachyura). Bulletin of Environmental Contamination and Toxicology (47) 4: 634-640.

Sokal RR \& RJ Rohlf. 1981. Biometry. W.H. Freeman and Company. Nueva York. EUA, 859 pp.

Vinuesa JH, GA Lovrich \& LI Comoglio. 1989. Temperature - salinity effects on the larvae development in false southern king crab, Paralomis granulosa, (Crustacea, Anomura). Thalassas 7: 53-57.

Ward GS \& PR Parrish. 1982. Manual of methods in aquatic environment research. Part 6. Toxicity. Test. FAO. Fisheries Technical Papers 185: 23 pp.

Weis JS, A Cristini \& K Ranga Rao. 1992. Effects of pollutants on molting and regeneration in Crustacea. American Zoologist 32: 495-500.

Wells PG \& JB Sprague. 1976. Effects of crude oil on American lobster (Homarus americanus), larvae in the laboratory. Journal of the Fisheries Research Board of Canada 33: 1604-1614. 